\title{
Aplicación móvil para la optimización de procesos de consumo de energía en sector hotelero
}

\author{
Mobile application for the optimization of energy consumption processes in the hotel \\ sector
}

ROJAS-NANDO, Julio Cesar†*, REYES-LUNA, Nayely y MARTINEZ-SALAZAR, Diego

Instituto Tecnológico Superior de Acatlán de Osorio

ID $1^{\text {er }}$ Autor: Julio Cesar, Rojas-Nando / ORC ID: 0000-0002-1143-3982, CVU CONACYT ID: 453986

ID $1^{\text {er }}$ Coautor: Nayely, Reyes-Luna / ORC ID: 0000-0001-5296-5427, CVU CONACYT ID: 680753

ID $2^{\text {do }}$ Coautor: Diego, Martinez-Salazar / ORC ID: 0000-0002-1488-632X, CVU CONACYT ID: 1000379

DOI: $10.35429 / J I T C .2019 .10 .3 .7 .12$

Recibido 06 de Junio, 2019; Aceptado 25 de Noviembre, 2019

\begin{abstract}
Resumen
La ingeniería es el conjunto de conocimientos tecnológicos que ayudan en la innovación, invención, desarrollo y mejora de técnicas y herramientas que satisfacen las necesidades y resuelven problemas de empresas y la sociedad en general, creando proyectos que facilitan la solución a cualquier problemática planteada, no importa de qué índole esta sea, siempre visualizando el beneficio apegado a una sustentabilidad en general. Al paso de los años y con el crecimiento demográfico se visualiza que se tiene el consumo excesivo de los recursos naturales, se necesita dar soluciones acertadas que poco a poco vayan minimizando toda esta situación. En este proyecto se visualizó realizar una aplicación Móvil llamada Applight, la cual ayudara a disminuir el consumo de energía en hoteles, a través de un indicador que nos avisara si algún electrodoméstico o lámparas y focos quedaron activados, teniendo así la oportunidad de poder apagar y desactivar todos aquellos componentes que al salir quedaron prendidos. De esta manera se ayudara a reducir el consumo de energía, en gran cantidad así se contribuye al cuidado del medio ambiente y al gasto de pagos de energía en las empresas hoteleras.
\end{abstract}

Sustentable, Aplicación , Móvil

\begin{abstract}
Engineering is the set of technological knowledge that helps in innovation, invention, development and improvement of techniques and tools that meet the needs and solve problems of companies and society in general, creating projects that facilitate the solution to any problem raised, not It matters what nature it is, always visualizing the benefit attached to a sustainability in general. Nowadays the biggest problem we have is the excessive consumption of natural resources, we need to give sound solutions that little by little will minimize this situation. In this project, a mobile application called Applight was visualized, which will help to reduce energy consumption in hotels, through an indicator that will let us know if any household appliance or lamps and lights were activated, thus having the opportunity to turn off and deactivate all those components that were left on when leaving. In this way, it will help to reduce energy consumption, in a large amount thus contributing to the care of the environment and the expenditure of energy payments in hotel companies.
\end{abstract}

Sustainable, Application, Mobil

Citación: ROJAS-NANDO, Julio Cesar, REYES-LUNA, Nayely y MARTINEZ-SALAZAR, Diego. Aplicación móvil para la optimización de procesos de consumo de energía en sector hotelero. Revista de Tecnologías de la Información y Comunicaciones. 2019. 3-10: 7-12

\footnotetext{
* Correspondencia del Autor (Correo electrónico: jc.rojas@hotmail.com)

$\dagger$ Investigador contribuyendo como primer autor.
} 


\section{Introducción}

En la actualidad a nivel mundial las inclemencias del clima y las consecuencias del exceso de consumo de los recursos naturales están marcando la vida diaria de todas las personas, afectando en gran medida la actividad de cada uno (Estrella Suárez \& González Vázquez , 2014). Las grandes industrias y empresas consumen un sinfín de recursos tanto energéticos como de otro tipo, provocando así contaminación y calor al ambiente en una forma excesiva (PRODECEN, 2018).

Son muchas las soluciones en las que se han pensado para contrarrestar esta problemática, y pocas las empresas e industrias que lo aplican, no obstante se sigue concientizando y generando posibles soluciones, para solventar el problema que cada día crece más (Nuñez, 2016). Dentro de las muchas alternativas de solución tenemos la utilización de energías renovables, el horario de verano, entre otros. El problema es mayúsculo ya que casi en su totalidad los dispositivos que existen, necesitan de energía eléctrica para poder funcionar (PRODECEN, 2018).

Las tecnologías de la información y comunicación (TIC's) también ofrecen alternativas de reducción de consumo de energía eléctrica, a través de aplicaciones que ayuden a realizar una utilización eficaz de la energía dentro de las empresas (Villaplana, 2017). Como es el caso de Applight, ya que de acuerdo a los diferentes hallazgos que se presentan en el consumo excesivo de energía dentro del sector hotelero se visualiza una oportunidad para mejorar los impactos tanto en la parte ambiental como económica del mismo (MOLINA, 2014). Promoviendo así la concientización del uso y manejo adecuado de la energía (Nuñez, 2016).

La realización de esta aplicación tiene como finalidad poder contribuir a la reducción de consumo eléctrico en el sector hotelero, el poder y tener y llevar un consumo responsable de los recursos naturales es importante para la buena sustentabilidad que se necesita en el planeta. Según el programa de las Naciones Unidas para el Desarrollo nos dice que el solo el $3 \%$ del agua del mundo es utilizable para beneficio del ser humano y la consume más rápido de lo que la naturaleza demora en reponerla.
Es por ese motivo que se pretende contribuir con este proyecto al cuidado de nuestros recursos naturales, proporcionando una solución dentro de un sector que se considera de alto consumo de energía (Molina, 2014).

La aplicación Applight, tiene un impacto determinante en el sector hotelero, que es el segmento de mercado donde se aplica, puesto que revoluciona la reducción del consumo de energía eléctrica en cada una de las habitaciones con las que cuente cada uno de los hoteles donde se manipula, ya que tendrá la capacidad de minimizar y controlar el manejo excesivo de la energía.

Con el uso de Applight se pretende mejorar la economía del sector hotelero, así como contribuir al cuidado del medio ambiente (MOLINA, 2014).

¿Cuál es su valor agregado respecto de las demás técnicas?

Existen diferentes aplicaciones las cuales utilizan la misma tecnología y método pero con algunas diferencias considerables:

\section{- $\quad$ Bajo costo \\ - Mejor Rendimiento \\ - Usabilidad \\ - $\quad$ Fácil interacción con el usurario. \\ - Mantenimiento accesible.}

Finalmente Applight, es una aplicación de fácil manejo con una interfaz amigable, para que pueda ser manipulada de una forma correcta por los usuarios, tiene un alcance de conexión bastante eficaz para su buen desempeño, facilita el poder reducir de una forma automatizada el consumo de energía por descuido al dejar prendidas las luces o aparatos (PRODECEN, 2018). Applight soluciona una de tantas problemáticas de una manera segura y adecuada al poder tener control del uso de la energía no renovable como lo es la energía eléctrica (Nuñez, 2016).

\section{Aplicación de herramientas}

En este apartado se pretende administrar información sobre las herramientas utilizadas para el proceso o desarrollo del proyecto, aplicando los métodos que garanticen la realización del proyecto y se puedan realizar pruebas de una forma eficaz y con resultados óptimos.

ROJAS-NANDO, Julio Cesar, REYES-LUNA, Nayely y MARTINEZ SALAZAR, Diego. Aplicación móvil para la optimización de procesos de consumo de energía en sector hotelero. Revista de Tecnologías de la Información y Comunicaciones. 2019 
Herramientas para el desarrollo:

\section{- $\quad$ MIT App Inventor:}

App Inventor es un lenguaje de programación basado en bloques (como piezas de un juego de construcción), y orientado a eventos. Sirve para indicarle al "cerebro" del dispositivo móvil qué queremos que haga, y cómo. Es un entorno de desarrollo de software creado por Google Labs para la elaboración de aplicaciones destinadas al sistema operativo Android. El usuario puede, de manera visual y a partir un conjunto de herramienta básica, enlazar dichos bloques para crear la aplicación. (Creative Commons Atribucion 4.0 Internacional APP INVENTOR, 2015)

\section{- $\quad$ Arduino uno 1.8.10 (El IDE de Arduino):}

Es un entorno de programación que ha sido empaquetado como un programa de aplicación, consiste en un editor de código, un compilador, un depurador y un constructor de interfaz gráfica (GUI).

El uso de recursos utilizado:

Laptop: Es una computadora portátil de peso y tamaño ligero, su tamaño es aproximado de un portafolio (hay más pequeñas como Palmtop y Handheld). Ésta pertenece en grupo de computadora personal.

Arduino: Es una placa electrónica basada en el microcontrolador ATmega328. Cuenta con 14 entradas/salidas digitales, de las cuales 6 se pueden utilizar como salidas PWM (Modulación por ancho de pulsos) y otras 6 son entradas analógicas.

Protoboard: Es una placa que posee unos orificios conectado eléctricamente entre sí siguiendo un patrón horizontal o vertical.

\section{Ingeniería del proyecto}

La ingeniería es el conjunto de conocimientos tecnológicos para la innovación, invención, desarrollo y mejora de técnicas y herramientas para satisfacer las necesidades y resolver problemas de empresas y la sociedad para crear proyectos, en donde a través de una lluvia de ideas se aterrizó una solución a la problemática que en nuestro caso lo denominamos "Applight" el cual está dirigido para negocios de hoteles.
Donde se diseñó un protoboard con ayuda del arduino el cual es parte esencial de la aplicación, como se muestra en la fig. 1.

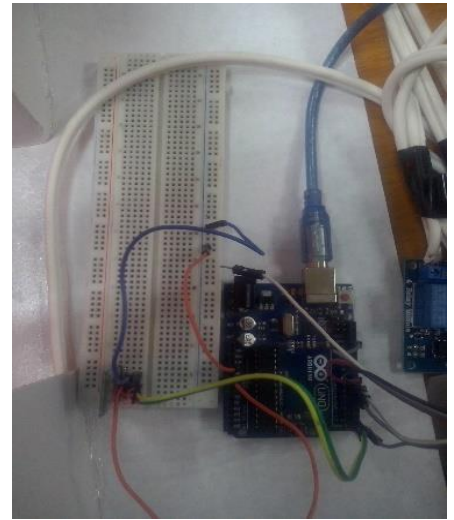

Figura 1 Diseño del Protoboard con el Arduino

La aplicación móvil se realizó para 20 habitaciones de un hotel, la simulación se demostró en una maqueta donde representa una habitación, la cual contiene todos los servicios que manipula la aplicación, como se muestra en la figura 2.

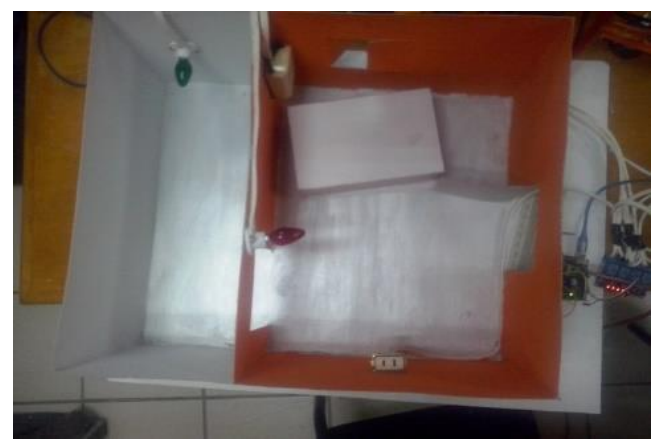

Figura 2 Maqueta de simulación de los cuartos del hotel

Esta manipulación se realizó mediante una conexión vía bluetooth, a través del cual se busca el dispositivo a manipular, figura. 3. Una vez localizado el dispositivo nos envia una nueva interfaz, la cual indica apagar los focos y electrodomésticos que controla un administrador, figura 4 .

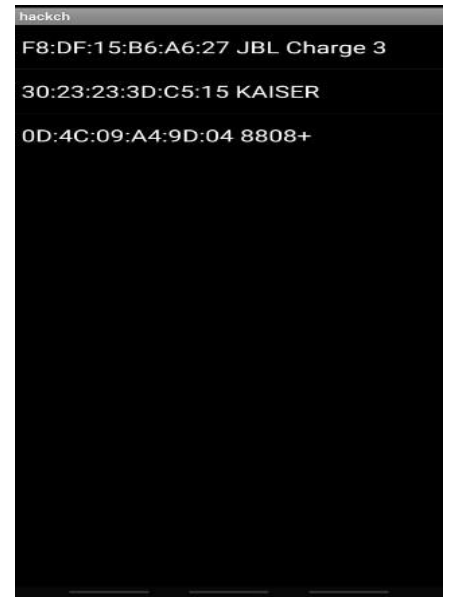

Figura 3 Conexión del bluetooth con el dispositivo

ROJAS-NANDO, Julio Cesar, REYES-LUNA, Nayely y MARTINEZSALAZAR, Diego. Aplicación móvil para la optimización de procesos de consumo de energía en sector hotelero. Revista de Tecnologías de la Información y Comunicaciones. 2019 


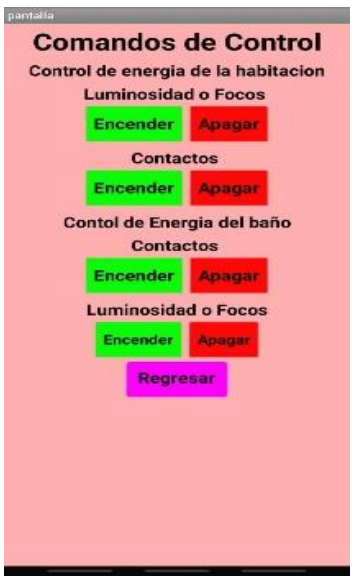

Figura 4 Interfaz de control de apagado

Esta conexión se administra y controla con la aplicación móvil Applight, la cual tiene la funcionalidad de poder emitir alarmas mediante las cuales avise o indique cuando un aparato o un foco dentro de una habitación han quedado encendidos y así mismo estos puedan ser desactivados desde el lugar donde se encuentre el usuario de ese cuarto, a través del celular y con ayuda de esta aplicación.

Se construyó una aplicación interactiva y amigable con el usuario final, que fuera de fácil manipulación con la finalidad de poder abarcar la mayoría del mercado y ser de gran aceptación para el cliente.

Como se puede visualizar en la Figura 5., la aplicación cuenta con un inicio de sesión, la cual permitirá al gerente tener un control más eficaz y eficiente del consumo y reducción de energía eléctrica.

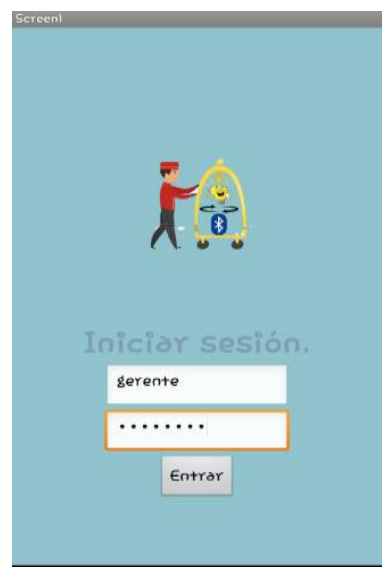

Figura 5 Interfaz de inicio de sesión
Posterior al inicio de sesión se muestra un menú, en el cual la interfaz permite seleccionar la opción que se desea verificar y controlar. Tal y como se presenta en la figura 6 .

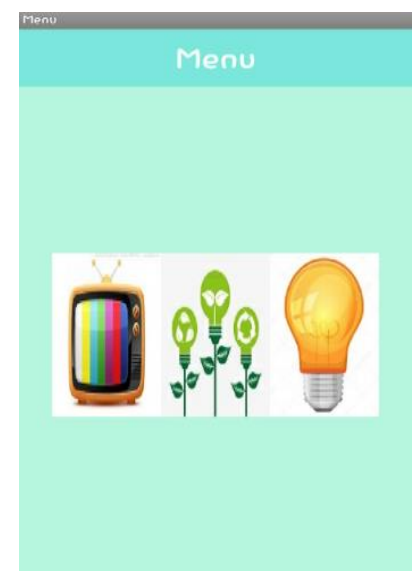

Figura 6 Interfaz menú

Finalmente podemos visualizar de una manera real, la forma y colocación del dispositivo que se conecta a través de Bluetooh con el celular y de esa manera manipular cada una de las acciones que se programaron figura 7.

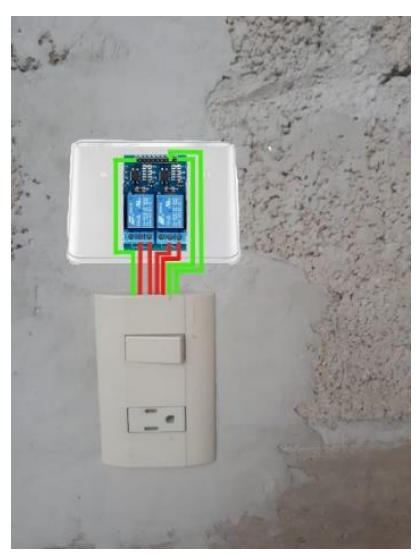

Figura 7 Placa o dispositivo real de conectividad

\section{Modelo de negocios}

El modelo de negocio que se utilizó para esta aplicación, fue el modelo Canvas, ya que es muy bueno para empezar a analizar los elementos que forman parte del proyecto. Este modelo parte del análisis del valor de la idea, es decir cuál es la finalidad del proyecto y cuál es su valor innovador, así mismo enlaza el análisis del cliente sus necesidades que cubre, los beneficios y la forma en cómo se va a distribuir. (ACF International, s.f.) 
Para Applight, se estableció el modelo de negocios, donde se señala la propuesta de valor, la relación que se tiene con los clientes, el segmento que se abarcara, así como los recursos que se necesitan para su difusión, entre algunos otros que se muestran en la tabla 1.

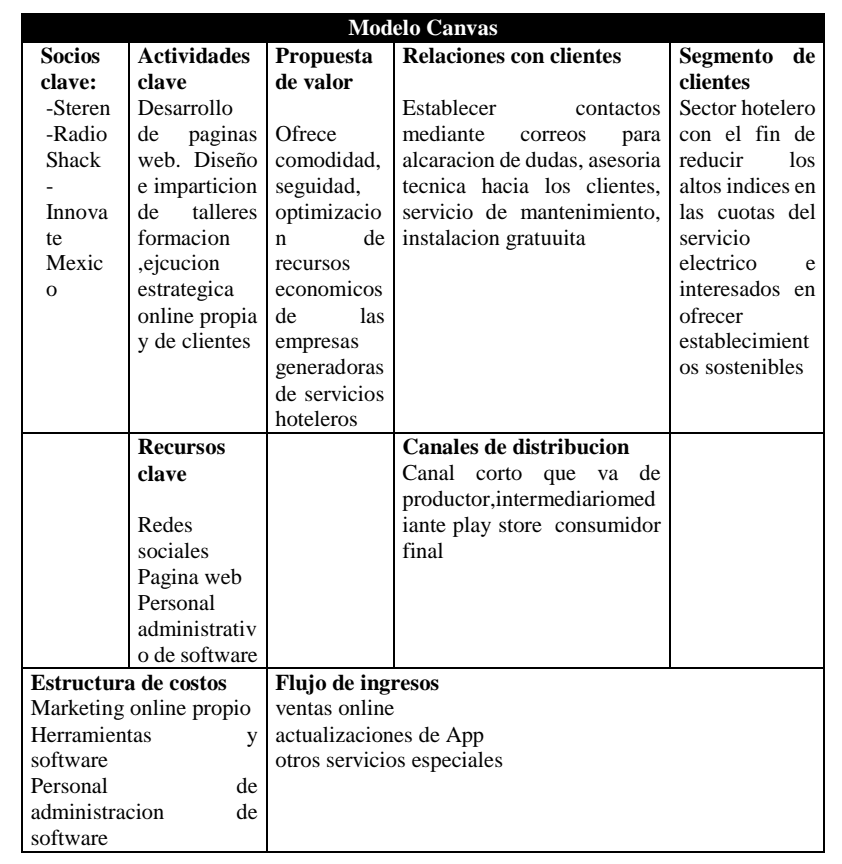

Tabla 1 Modelo Canvas

\section{Análisis de factibilidad, alcance y proyección}

Con base al análisis económico realizado el proyecto se obtuvo una TMAR de $28.36 \%$ y una TIR 59.5\% de acuerdo al criterio para la toma de decisiones que el autor afirma es:

- $\quad$ Si TIR TMAR < TIR es recomendable aceptar la inversión

Si TMAR > TIR es preciso rechazar la inversión (Urbina, 2007).

Por lo que es recomendable efectuar la inversión. Una vez realizado el análisis de costo de acuerdo a la implementación del proyecto APPLIGHT mediante la fórmula del valor presente neto se obtiene una utilidad de $\$ 1$, $605,798.46$ del porcentaje de ganancia que se ha fijado como mínima aceptable, es decir una tasa mínima aceptable de rendimiento del $28.36 \%$

\section{Metodología a desarrollar}

Para fines de este proyecto se utilizó la metodología de desarrollo de software para sistemas dinámicos, (DSDM). DSDM es un marco comprobado para la gestión y entrega de proyectos Agiles, que ayuda a entregar resultados de manera rápida y eficaz.
A lo largo de los años, se ha aplicado a una amplia gama de proyectos, desde pequeños desarrollos de software hasta cambios de procesos empresariales a gran escala. (Agile Business Consortium, 2019) A pesar de que DSDM funciona de manera fácil y efectiva en proyectos pequeños y simples, siempre ha mantenido un fuerte enfoque en el entorno corporativo basado en proyectos para proporcionar un enfoque "adulto" a Ágil en el complejo mundo corporativo. Su meta es entregar los sistemas del software a tiempo y en el presupuesto mientras es ajustando para los requisitos cambiantes a lo largo del proceso de desarrollo.

Esta metodología está dividida en tres fases importantes:

\section{- $\quad$ Fase del pre-proyecto, \\ - $\quad$ Fase del ciclo de vida del proyecto \\ - $\quad$ Fase del post-proyecto} construir.

Fase 1: Se selecciona el proyecto a Se determina los casos de estudios:

- Visión General del sistema.

- Se determinan alcances del sistema.

- Características funcionales y técnicas.

- $\quad$ Estudio de viabilidad. aplicación:

Fase 2: Se determina los diseños de la

1. Estudio de viabilidad: Se evalúa si la aplicación es viable, para el proceso teniendo en cuenta los requisitos básicos del negocio y sus restricciones asociadas.

2. Estudio de la empresa: Se establecen los requisitos funcionales $y$ de la información con el fin de permitir un valor al negocio; Además se define la arquitectura básica de la aplicación.

3. Iteración del modelo funcional, Todos los prototipos del MDSD están diseñados para evolucionar hacia la aplicación entregable.

4. Diseño e iteración de la estructura: Se revisa la construcción de prototipos durante la iteración del modelo funcional. En algunas ocasiones la iteración de construcción de diseño suceden de forma concurrente. 
5. Implementación: Se entrega una versión del sistema con el fin de capacitar al usuario y evaluar detalladamente los documentos de sistema.

- $\quad$ Diseño simple y ligero.

- $\quad$ Técnicas de modelado.

- Codificación de MIT app Inventor y prototipo del proyecto.

Pruebas del prototipo.

Fase 3: Sistema realizado y en funcionamiento. Dar el significado de las variables en redacción lineal y es importante la comparación de los criterios usados. Ver Figura 8 (Scrum Manager Body of Knowledge, 2012)

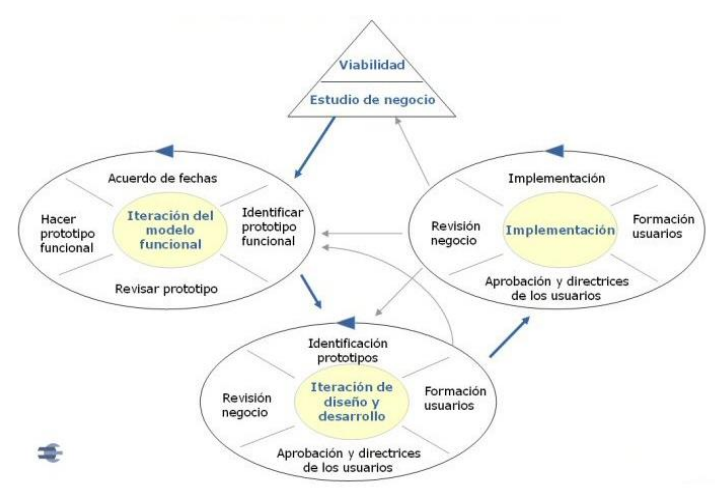

Figura 8 Diagrama de procesos de la metodología DSDM

\section{Resultados}

De acuerdo a los diferentes hallazgos que se presentan en el consumo de energía dentro del sector hotelero se presenta una oportunidad para mejorar los impactos obtenidos tanto en la parte ambiental como económica del mismo, mediante el uso de nuevas tecnologías que ayuden a minimizar y control el uso eficiente de este recurso, así como también la concientización del uso y manejo adecuado de la energía

\section{Conclusiones}

Actualmente el desarrollo de software para dispositivos móviles evoluciona día a día, lo que nos permite implementar diversas soluciones en la mayoría de los problemas actuales, el bajo costo y la gran cantidad de dispositivos hace amplia la aplicabilidad de estos, por lo tanto nos son de mucha utilidad tanto para aminorar costos así como de implementar soluciones que cuiden el medio ambiente

\section{Referencias}

ACF International. (s.f.). $\quad w w w$. accioncontraelhambre.org. Obtenido de https://www.accioncontraelhambre.org/sites/def ault/files/documents/pdf/explicacion_metologia _canvas.pdf

Agile Business Consortium. (2019). www.agilebusiness.org. Obtenido de https://www.agilebusiness.org/content/introduct ion-0

Bordigno, F., \& A. Iglesias, A. (2015). Diseño y Construccion de Objetos Interactivos Digitales. Buenos Aires, Argentina: Universitaria.

Creative Commons Atribucion 4.0 Internacional APP INVENTOR. (2015). www.codeweek.eu. Obtenido de https://codeweek.eu/docs/spain/guia-iniciacionapp-inventor.pdf

Estrella Suárez, M., \& González Vázquez , A. (2014). Desarrollo Sustentable, Un nuevi mañana. México: Patria.

Floyd, T. L. (2008). Dispositivos Electronicos. Mexico: Pearson Educación.

Molina, C. M. (2014). Eficiencia Energetica en las Empresas del Ramo de la Hoteleria. Turismo Sustentable, 4.

MOLINA, C. M. (2014). Efiecienfia Energética en las Empresas del Ramo de la Hoteleria. Turismo Sustentable II, 4.

Nuñez, A. J. (2016). Consumo de Energia, Desarrollo Sostenible y Ambiente. Centro de Estudios en Energía y Ambiente, FCFM, 69.

PRODECEN. (2018). Programa de Desarrollo del Sistema Electrico Nacional . México.

Scrum Manager Body of Knowledge. (31 de Diciembre de 2012). www.scrummanager.net. Obtenido de https://www.scrummanager.net/bok/index.php?t itle=File:Dsdm.jpg

Villaplana, Á. C. (2017). Tecnologías para el desarrollo Sustentable. Filosofía Univ. Costa Rica, 14. 